\title{
MPPT Control Method of PV Based on subregional variable duty cycle
}

\author{
LI Bing1, ZHONG Li Jun1, WANG Gang1', TIAN Xiaojian²
}

\author{
1.College of Mechanical Engineering, BaiCheng Normal College, Baicheng, 137000, China
}

2. College of Electronic Science and Engineering, Jilin University, Changchun, 130000, China

Keywords : subregional variable duty cycle ; MPPT ; Photovoltaic power generation; power curve

\begin{abstract}
A new method is presented for variable duty cycle of photovoltaic subregional maximum power point tracking(MPPT).It has combined the advantages of the popular MPPT method ,In different areas of photovoltaic power generation using different duty cycle control method, Optimization of the target area using constant voltage method, Reached quickly determine the maximum power point (MPP) when light mutations.
\end{abstract}

\section{Introduction}

Photovoltaic power generation is an excellent clean energy, but there still exist some problems in the large-scale applications of photovoltaic power generation, such as that the input cost of photovoltaic power generation is high, while the utilization ratio is low. Thus to guarantee the maximum output of the photovoltaic system and to conduct maximum power point tracking on the photovoltaic system is of vital significance to the promotion of photovoltaic power generation. This paper focuses on the research of the MPPT control method of PV to guarantee that the photovoltaic system can quickly and effectively track its maximum power point. The goal of this MPPT control method research is to pursue the maximum electricity output according to the maximum power of the photovoltaic system. There are many MPPT control methods among which the Constant Voltage Tracking (CVT) method, the Perturbation and Observation ( $\mathrm{P} \& \mathrm{O})$ method, the Incremental Conductance (IncCond) method, and the Fuzzy Control method etc. are the most commonly used. However, each method has its advantages and disadvantages that the rapidity of the tracking method contradicts with its precision and that we should also consider the application conditions of different photovoltaic systems. Now some scholars proposed to combine the method of variable step length and P \& O method or the IncCond method; this combination can accelerate the optimizing speed, but it possesses the disadvantages of large vibration near the maximum power point, and systematic dependency which means that the selection of parameters depends upon a particular system. Based on the above, this paper proposes a modified MPPT control method which introduces the rapidity of the CVT method and the precision of the IncCond method, and combines the subregional variable duty cycle to optimize the photovoltaic array. To verify and analyze on basis of the setting up of the variable duty cycle $\Delta D$ and the time parameter of the power/hour $T_{\mathrm{a}}$, and verify the method proposed in this paper on the experimental platform.

\section{Improvement of the MPPT of Photovoltaic Cells}

\section{The Principle of Photovoltaic MPPT}

The photovoltaic array is in nature a power generation unit controlled by natural conditions, which means that the quantity of the electricity power it generates is limited by the natural conditions. Thus to control its generation output requires us to control the output voltage and output current to guarantee the maximum output. The power curve of the simulation model of photovoltaic output built by the simulation software PSCAD is as shown in Figure 1.

The (a) and (b) curve in Diagram 1 are the P-V and I-V curve of the photovoltaic array within same temperature range but different illuminations $\left(500 \mathrm{w} / \mathrm{m}^{2}, 800 \mathrm{w} / \mathrm{m}^{2}, 1000 \mathrm{w} / \mathrm{m}^{2}\right)$

The PV system diagram (including BOOST Circuit Diagram) is as shown in Figure 2. In 
Figure 2, $\mathrm{U}_{\mathrm{s}}$ refers to Photovoltaic Power, $\mathrm{R}_{\mathrm{s}}$ refers Equivalent Resistance and the part in the dashed box marked 2 represents the BOOST Circuit Load Structure which is an equivalence to the $\mathrm{R}_{0}$ in Figure 3.

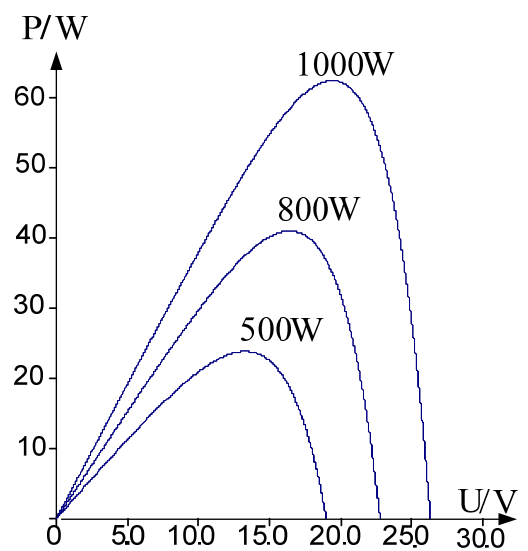

(a)

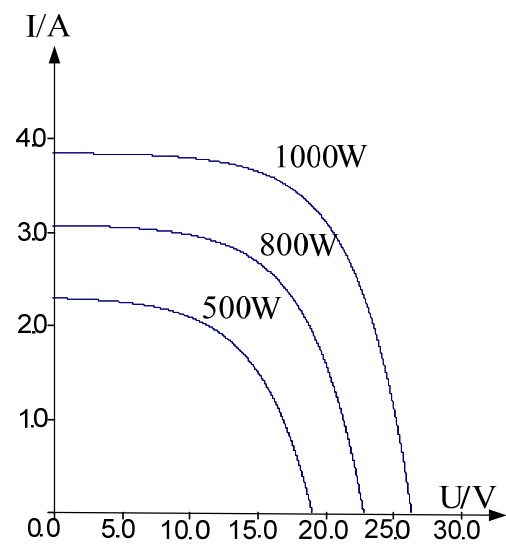

(b)

Fig. 1 The $P-V$ and $I-V$ curves in different illuminations

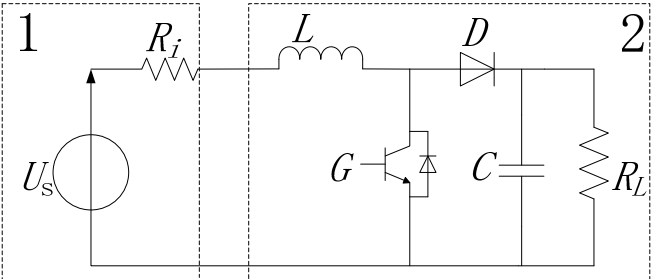

Fig.2 PV system diagram

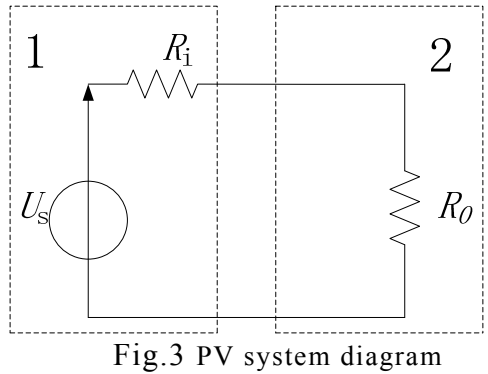

As we all know, the output power of the photovoltaic system can reach the maximum point in case of impedance matching. Therefore, the process of tracking the MPP of the photovoltaic array is, in effect, the process of constantly changing the Duty Cycle D to match $R_{0}$ and $R_{i}$. The photovoltaic array connects the BOOST circuit structure to output power and analyze small signal model, we can get the conclusion that the variation $(\triangle P)$ of the Output Power of the photovoltaic array $\left(P_{\mathrm{pv}}\right)$, and the duty cycle variation $(\Delta D)$ of the BOOST circuit satisfy the following approximate relationship: 


$$
\Delta P=-\frac{\Delta U_{\mathrm{pv}}{ }^{2}}{R_{\mathrm{MPP}}} \approx-\frac{\mu^{2} \Delta D^{2}}{R_{\mathrm{MPP}}} f\left(T_{\mathrm{a}}\right)
$$

$f\left(T_{a}\right)$ is the function of the sampling time $T_{a}$, and $\mu$ is the opposite number of the output power $\left(U_{\mathrm{o}}\right)$ of the Boost circuit.

Analyzing from the above, we can conclude that we can dynamically change $R_{0}$ through dynamically changing $\Delta D$ to achieve impedance matching, and thus to achieve the maximum dynamic output power. Compared with the traditional method, this method saves the process of voltage/current control loop, thus greatly simplifying the control process. MPPT is in essence the dynamic process of real-time comparison, real-time computing and real-time output.

\section{Improving Method of the MPPT of the Variable Duty Cycle}

Among the existing methods, there exist a contradiction between the rapidity and the vibration output loss in the MPPT of PV. A greater duty cycle with better rapidity means inability to work at a stable power point, while a smaller duty cycle means lower rapidity, poor dynamic effect and unsatisfactory function in acute illumination change which is very common in field application.

Therefore, this paper proposes a MPPT control method based on subregional variable duty cycle. On basis of the rapid algorithm of the variable duty cycle, this control method adopts variable $\Delta D$ through taking the extension area of the CVT as its regional boundary to achieve MPPT. The subreginal schematic diagram is as shown in Figure 4.

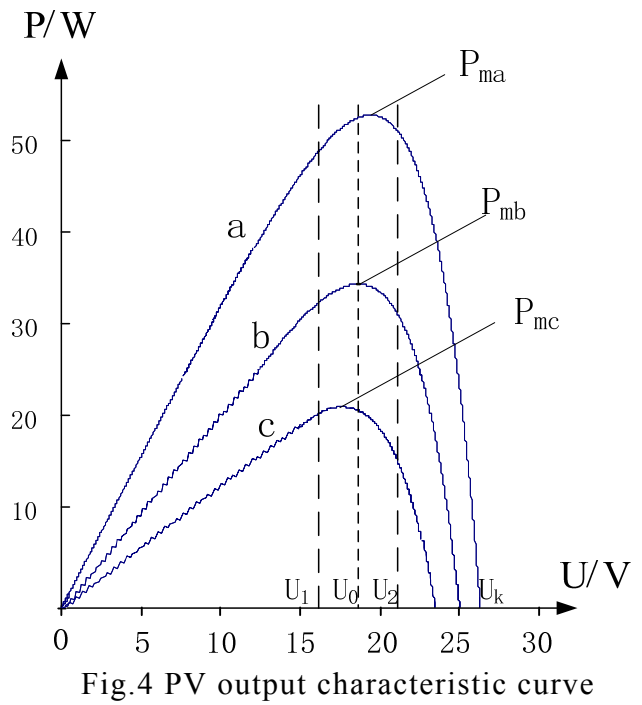

Set a targeted region: $U_{1}-U_{2}$, and the point in this region is marked as $U_{0} U_{0}$ in this region represents the corresponding voltage of the maximum power point $P_{\mathrm{m}}$ set in the CVT method. On basis of our field measuring data, we set the length of $U_{1}-U_{0}$ and $U_{1}-U_{0}$ as $20 \% U_{k}, U_{k}$ representing the Open-circuit Voltage. $\Delta D$ in $0-U_{1} 、 U_{2}-U_{\mathrm{k}}$ is the great duty cycle and in $U_{1}-U_{2}$ is small duty cycle.

The influence of the value of $\Delta D$ on the MPPT will be firstly analyzed through simulation, selecting proper value for $\Delta D$ and $T_{\mathrm{a}}$. Figure 5 shows the two duty cycle curves $(D=0.05$ and $D=0.005)$ for simulated analysis under the PSCAD. From this diagram we can see that when $\mathrm{D}=0.05$, the optimizing speed is high, but the vibration is comparatively great; when $\mathrm{D}=0.005$, the optimizing effect is just the other way around. This paper proposes to respectively carry out MPPT under the circumstance of the two variable $\Delta D$ values. 


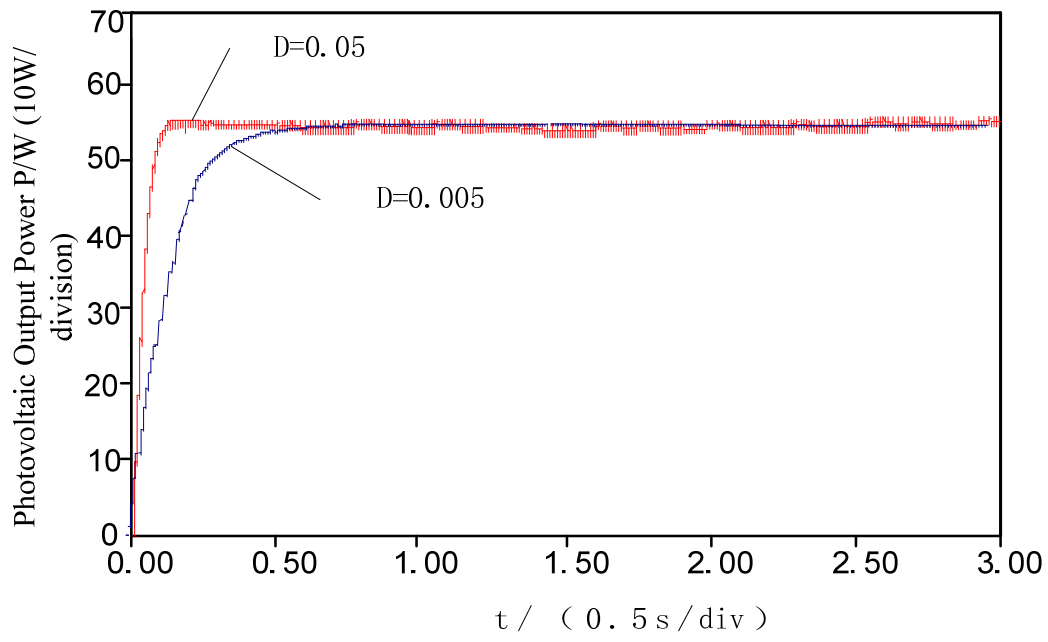

Fig. 5 different duty cycles comparison chart

Similar to set of the duty cycle $\mathrm{D}$, the problem of rapidity and precision also exists in the set of the time constant $T_{\mathrm{a}}$. So synthetically consider, we set the time constant $T_{\mathrm{a}}=0.001$.

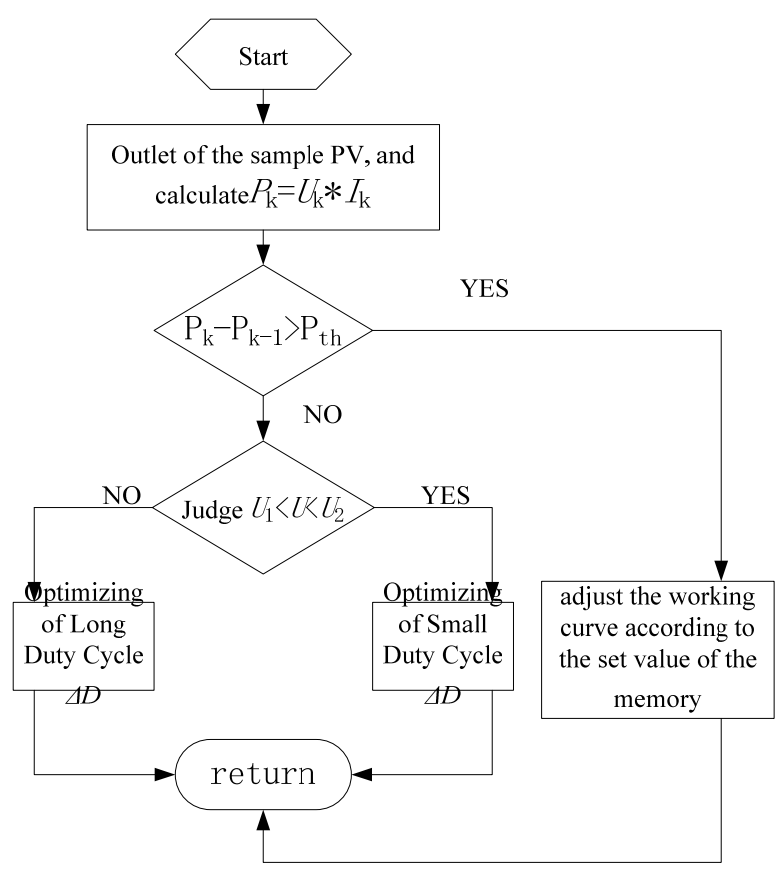

Fig.6 Control flow chart

When the illumination suddenly changes, the MPTT should have sound dynamic properties. Measure the illumination of a region and get the different MPPT curve of different illumination conditions, and then save them in the memory of the system. We can judge the working curve of the photovoltaic array in circumstance of sudden illumination change. When the illumination condition suddenly changes, set a power change value $\mathrm{P}_{\text {th }}$ and adjust the corresponding voltage value of the existing $P_{\mathrm{m}}$ to do MPPT of the small duty cycle $\Delta D$ near this value to reduce power vibration.

The control flow chart of the subreional variable duty cycle is as shown in Figure 6.

\section{Conclusion}

From the above analysis we can conclude that compared with the traditional tracking method, the MPPT control method based on subregional variable duty cycle has the advantage of rapid optimization and small vibration. The photovoltaic array can rapidly determine its optimizing point even in circumstances that the external abrupt illumination change has gone beyond the set value. 
Conducting MPPT near this point overcomes the problems of output misjudgment and great vibration near the maximum power point. Simple and precise and without extra investment for hardware, the method proposed in this paper is of great guiding significance to the promotion and application of photovoltaic popularization.

\section{Reference}

[1] Yishuang Geng, Jin Chen, Ruijun Fu, Guanqun Bao, Kaveh Pahlavan, Enlighten wearable physiological monitoring systems: On-body rf characteristics based human motion classification using a support vector machine, IEEE transactions on mobile computing, 1(1), 1-15, Apr. 2015

[2] Jie He, Yishuang Geng, Fei Liu, Cheng Xu, CC-KF: Enhanced TOA Performance in Multipath and NLOS Indoor Extreme Environment, IEEE Sensor Journal, 14(11), 3766-3774, Nov. 2014

[3] Jie He, Yishuang Geng, Kaveh Pahlavan, Toward Accurate Human Tracking: Modeling Time-of-Arrival for Wireless Wearable Sensors in Multipath Environment, IEEE Sensor Journal, 14(11), 3996-4006, Nov. 2014

[4] $\mathrm{Na} \mathrm{Lu}$, Caiwu Lu, Zhen Yang, Yishuang Geng, Modeling Framework for Mining Lifecycle Management, Journal of Networks, 9(3), 719-725, Jan. 2014 